\title{
Capillary Filling of Anodized Alumina Nanopore Arrays
}

\author{
Kyle J. Alvine, ${ }^{1, *, \dagger}$ Oleg G. Shpyrko, ${ }^{1,2}$ Peter S. Pershan, ${ }^{1,3}$ Kyusoon Shin, ${ }^{4,5}$ and Thomas P. Russell ${ }^{5}$ \\ ${ }^{1}$ Division of Engineering and Applied Sciences, Harvard University, Cambridge, Massachusetts 02138, USA \\ ${ }^{2}$ Center for Nanoscale Materials, Argonne National Laboratory, Argonne, Illinois 60439, USA \\ ${ }^{3}$ Department of Physics, Harvard University, Cambridge, Massachusetts 02138, USA \\ ${ }^{4}$ School of Chemical and Biological Engineering, Seoul National University, Seoul, Republic of Korea \\ ${ }^{5}$ Department of Polymer Science and Engineering, University of Massachusetts, Amherst, Massachusetts 01003, USA
}

(Received 26 July 2006; published 27 October 2006)

The filling behavior of a room temperature solvent, perfluoromethylcyclohexane, in approximately $20 \mathrm{~nm}$ nanoporous alumina membranes was investigated in situ with small angle x-ray scattering. Adsorption in the pores was controlled reversibly by varying the chemical potential between the sample and a liquid reservoir via a thermal offset, $\Delta T$. The system exhibited a pronounced hysteretic capillary filling transition as liquid was condensed into the nanopores. These results are compared with KelvinCohan theory, with a modified Derjaguin approximation, as well as with predictions by Cole and Saam.

DOI: 10.1103/PhysRevLett.97.175503

PACS numbers: 61.10.Eq, 05.70.-a, 68.08.Bc

Nanoporous materials hold great potential in a wide variety of nanotechnology applications such as DNA translocation [1,2], nanofluidic transistors [3], templates for nanoparticle self-assembly [4,5], catalysis [6], sensors for chemical agents [7], as well as more commonplace applications such as filtering and humidity sensors [8]. The breadth of nanopore research serves to underscore the need for a good physical understanding of the evolution of liquids in such systems. Previous wetting experiments and simulations used single pores with both wedgelike [9] and slitlike $[10,11]$ geometries, as well as complex porous networks, such as the disordered Vycor [12] and M41S silica materials [13]. Unfortunately, these porous glasses have very complicated network geometries that make it rather difficult to compare the measurements with simple theoretical predictions. Additionally, many of these studies are done at cryogenic temperatures that are impractical for all of the applications described above. Room temperature measurements on ordered nanoporous systems with nearly ideal geometry should provide insight into the physical processes governing behavior of liquids in confinement.

We describe here in situ small angle x-ray scattering (SAXS) experiments of the equilibrium wetting and capillary condensation of a room temperature solvent, perfluoromethylcyclohexane (PFMC), within nanoporous alumina $\left(\mathrm{Al}_{2} \mathrm{O}_{3}\right)$. The anodized alumina system had an ideal geometry described by a parallel arrangement of cylindrical nanopores (diameter $\sim 20 \mathrm{~nm}$ ) with large aspect ratios $\sim 1: 5000$ (see Fig. 1) [14]. We carried out experiments within an environmental chamber that allowed precise control of the amount of solvent condensed within the pores via changes in the chemical potential offset, $\Delta \mu$, relative to liquid/vapor coexistence similar to studies of wetting on flat [15] and nanostructured surfaces [16]. Both adsorption and desorption processes were investigated reproducibly via this technique.
Porous alumina membranes were prepared electrochemically using a two-step anodization technique $[14,17]$ and cleaned as described elsewhere [4]. The resultant alumina membrane consisted of an array of cylindrical parallel pores running the entire thickness of the membrane and open on both ends. The macroscopic dimensions of the nanoporous membrane were about $1 \mathrm{~cm} \times 1 \mathrm{~cm} \times 90 \mu \mathrm{m}$. Pores formed a 2D locally ordered hexagonally close-packed structure with nearest neighbor distances (center to center) of $58 \pm 4 \mathrm{~nm}$, and diameter of $21 \pm 5 \mathrm{~nm}$ as determined via electron microscopy (see Fig. 1). After cleaning, samples were dried and loaded into a hermetically sealed environmental chamber under an atmosphere of "ultrapure" grade $\mathrm{N}_{2}$.

The environmental chamber as described elsewhere $[4,15,18]$ consisted of two concentric cylindrical metal chambers that allowed extremely stable (drift of $<5 \mathrm{mK} / \mathrm{hr}$ ) temperature control of the sample to within

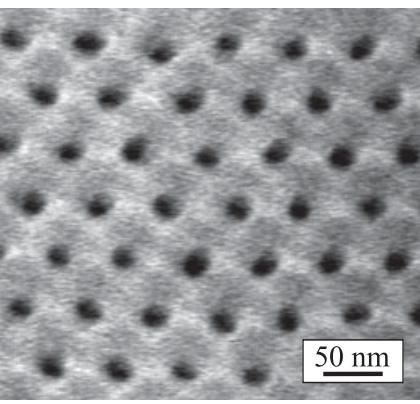

(a)

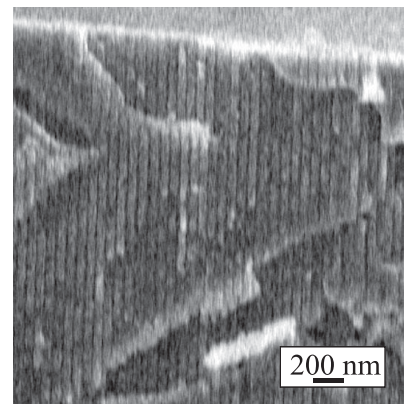

(b)
FIG. 1. (a) SEM image shows well ordered hexagonal packing of alumina nanopores. Reprinted with permission from our previously published work [4]. Copyright by the American Physical Society. (b) SEM of the fractured edge of the membrane showing the parallel packing of the nanopore channels (seen as vertical lines) normal to the membrane surface. 
a readout precision of $1 \mathrm{mK}$. Condensation of liquid solvent into the pores was precisely controlled via a positive offset $\Delta T$ between the sample temperature, $T_{s}$, and the temperature, $T_{r}$, of a liquid PFMC (purum grade, 97\% GC) reservoir, related to the chemical potential offset by $\Delta \mu \approx$ $H_{\text {vap }} \Delta T / T_{r}[4,15,18]$. Here $H_{\text {vap }}=33.9 \mathrm{~kJ} / \mathrm{mol}$ is the heat of vaporization of PFMC. [19] For large $\Delta T$, little or no liquid condenses in the pores. As the $\Delta T$ (and thus $\Delta \mu)$ is decreased toward zero, increasing amounts of liquid condense into the pores until saturation (complete volume filling) is obtained.

During the experiment, the inner and outer chambers were set at $T_{\text {in }}=31.6{ }^{\circ} \mathrm{C}\left(T_{r}=31.9{ }^{\circ} \mathrm{C}\right)$ and $T_{\text {out }}=$ $28.5^{\circ} \mathrm{C}$, respectively. Although the sample was nearly thermally isolated from the inner chamber, for high sample temperatures the large heat loads on the sample increased $T_{\text {in }}$ and $T_{r}$ to $36^{\circ} \mathrm{C}$ and $38^{\circ} \mathrm{C}$, respectively. Stability of $T_{r}$ was better than $50 \mathrm{mK}$ throughout the experiment.

In situ small angle $\mathrm{x}$-ray scattering (SAXS) data were collected using incident monochromatic $\mathrm{Cu}-K_{\alpha 1}$ radiation with high horizontal angular resolution of $0.005^{\circ}$ (87 $\mu \mathrm{rad}$ ) achieved using a triple-bounce channel cut Bonse-Hart [20] Ge(111) monochromator. High horizontal detector resolution was obtained by mounting an identical triple-bounce $\mathrm{Ge}(111)$ analyzer crystal after the sample, in the nondispersive geometry [21] (see Fig. 2). Scattered photons were collected with a scintillation detector.

The alumina membrane was mounted in a transmission geometry with the face of the membrane nearly perpendicular to the $\mathrm{x}$-ray beam, aligning the long axis of the pores nearly parallel to the beam. In order to take advantage of the high horizontal angular resolution, the mem-

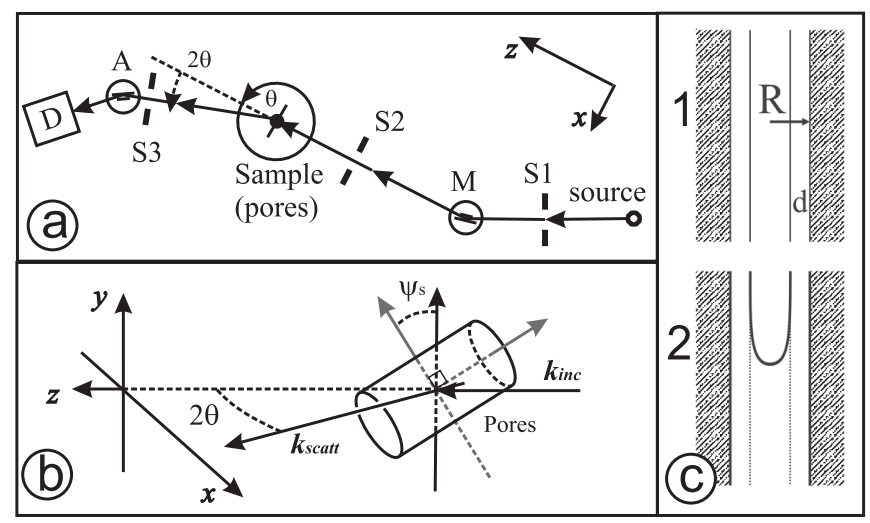

FIG. 2. SAXS Geometry: (a) Spectrometer setup: A triplebounce monochromator $(M)$ selects $\mathrm{Cu} K_{\alpha 1}$ radiation with high angular resolution in the horizontal plane. An identical triple-bounce analyzer crystal (A) is used after the sample, before the detector (D). (b) Alumina pores are tilted, $\psi_{s}=$ $8.5^{\circ}$, in the $y-z$ plane away from the parallel condition to take advantage of the enhanced horizontal resolution. Scattered intensity is measured as a function of $2 \theta$ in the $x-z$ plane. (c)(1) Conformal pore filling with film thickness, $d$. (c)(2) Partially filled (metastable condition) pore with meniscus during desorption. brane was tilted in the vertical direction by $\psi_{s} \approx 8.5^{\circ}$. This tilt effectively confined the scattering to the horizontal plane and minimized waveguide or multiple scattering effects due to grazing angle reflections from the pore walls. In this geometry, the intensity of the $\langle 10\rangle$ powder diffraction peak, due to the 2D hexagonal packing arrangement of the coaligned pores with a well defined pore-pore distance, was recorded in situ as $\Delta T$ was varied between $0.1 \mathrm{~K}$ up to $27 \mathrm{~K}$ for both adsorption (cooling) and desorption (heating) paths.

Immediately prior to collecting the data presented here, the pores were cleaned by repeated "flushing" (produced by rapid thermal cycling over the full $\Delta T$ range) with PFMC over ten thermal cycles. The intensity of the $\langle 10\rangle$ diffraction peak recorded at the highest $\Delta T=27 \mathrm{~K}$ gradually increased over the first three flushing cycles and remained unchanged during subsequent cycles.

As liquid condenses into the pores, replacing $\mathrm{N}_{2}$, the electron density contrast of the system decreases, eventually saturating when the pores are completely filled with liquid. This reduction in contrast is measured by a corresponding decrease in the scattering intensity of the powder diffraction peaks, $I_{\text {peak }}(\Delta T)$, relative to the dry peak intensity, $I_{\text {peak }}$ (dry) (see Fig. 3). Neglecting absorption, the added solvent volume fraction, $V(\Delta T)$, can be determined within the small angle approximation [4,16] from the scattering intensity of the lowest order peak, the $\langle 10\rangle$, as follows:

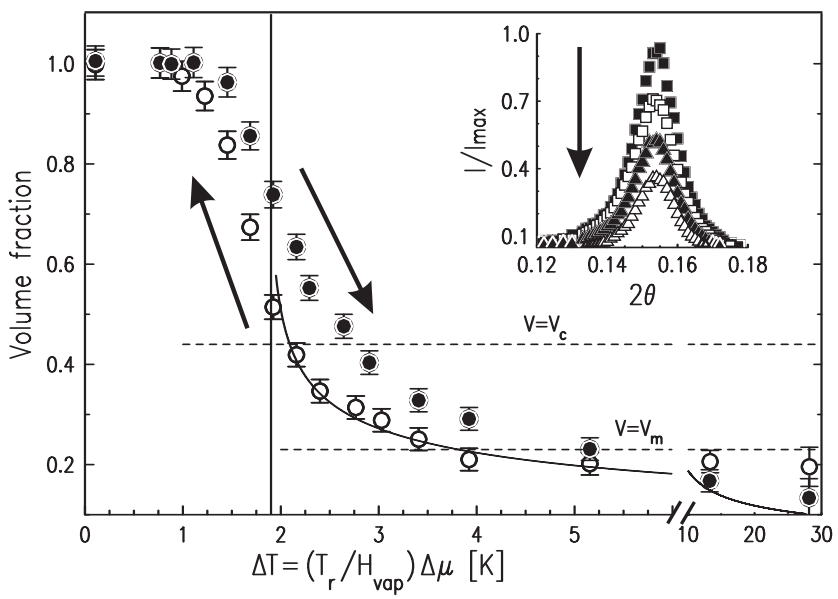

FIG. 3. Volume fraction as a function of $\Delta T$. Adsorption $(\bigcirc)$ and desorption (O) data are shown with arrows indicating the measurement direction. The horizontal axis scale is split $(/ /)$ in order to fit data at high $\Delta T$. A vertical line represents the Kelvin transition, $\Delta T=1.9 \pm 0.2 \mathrm{~K}$. The solid curve is a fit of the pretransition filling within the Derjaguin approximation. Horizontal dashed lines indicate the critical and metastable volume fractions, $V_{c}$ and $V_{m}$ respectively. Inset: The $\langle 10\rangle$ diffraction peak for various $\Delta T$ (symbols $\boldsymbol{\square}, \square, \boldsymbol{\Lambda}, \Delta$ correspond to $\Delta T$ of $9.9 \mathrm{~K}, 2.5 \mathrm{~K}, 2.0 \mathrm{~K}$, and $1.3 \mathrm{~K}$, respectively) during the absorption cycle. The arrow indicates the intensity decrease with decreasing $\Delta T$. 


$$
V(\Delta T) \propto \sqrt{I_{\text {peak }}(\text { dry })}-\sqrt{I_{\text {peak }}(\Delta T)}
$$

Because of van der Waals (vdW) interactions, the pores are, in practice, never "dry" or empty, making $I_{\text {peak }}$ (dry) difficult to determine. Even at the largest $\Delta T$ realized in this experiment, we still expect monolayer adsorption of material onto the walls of the pores, evidenced by the necessity of flushing. For small $\Delta T$, complete volume filling is easily observed by the intensity saturation. For the purposes of this analysis, we calculated a theoretical value for $I_{\text {peak }}$ (dry) from the ratio of the form factors squared for empty and PFMC filled pores. The form factor can be defined as a function of the radius, $R$, liquid film thickness, $d$, the electron densities, $\rho_{L}$ and $\rho_{S}$, for PFMC and $\mathrm{Al}_{2} \mathrm{O}_{3}$, respectively, and scattering vector $q=(2 \pi / \lambda) \sin (2 \theta)$ :

$$
\begin{aligned}
F\left(\rho_{L}, \rho_{S}, d, R\right) & =\frac{\rho_{L}}{q}\left[R J_{1}(q R)-(R-d) J_{1}(q(R-d))\right] \\
& -\frac{\rho_{S} R}{q} J_{1}(q R) .
\end{aligned}
$$

The ratio, $|F(d=R) / F(d=0)|^{2} \approx 0.3$, combined with the saturated intensity at low $\Delta T$, provided the necessary offset and normalization.

Values for the fractional volume condensed into the pores, derived from the $\langle 10\rangle$ diffraction peak intensity for both adsorption and desorption, are plotted in Fig. 3 as a function of $\Delta T$. X-ray adsorption corrections were applied to the highest $\Delta T$ measurements when increases in saturated vapor pressure of PFMC in the chamber were nonnegligible. The system exhibits a pronounced capillary transition upon filling, occurring at approximately $\Delta T^{*}=$ $1.9 \pm 0.2 \mathrm{~K}$ (at maximum slope, indicated with a solid vertical line in Fig. 3). Utilizing the simple Kelvin equation for capillary filling

$$
\Delta T^{*}=\left(\frac{T_{r}}{H_{\mathrm{vap}}}\right) \Delta \mu^{*}=\left(\frac{T_{r}}{H_{\mathrm{vap}}}\right) \frac{\gamma}{n R},
$$

where $\gamma=12.5 \mathrm{mN} / \mathrm{m}$ is the surface tension of the PFMC, and $n=5.1 \times 10^{3} \mathrm{~mol} / \mathrm{m}^{3}$ is the molar volume of the PFMC, this indicates a nanopore radius of $R=$ $11.8 \pm 1.0 \mathrm{~nm}$, in agreement with electron microscopy measurements. The system also exhibits hysteresis common to porous systems [22] with the desorption transition occurring at $\Delta T^{*}=2.3 \pm 0.2 \mathrm{~K}$. The width of this hysteresis is smaller by a factor of 5 than the prediction by Cohan [23] for a desorption transition at $2 \Delta T^{*}$ due to pores emptying by retreating menisci from the pore ends. Thus, the simple Cohan argument cannot provide a complete description of the hysteresis observed here, indicating the necessity of using a more complex theoretical approach.

In their seminal work, Cole and Saam [24,25] put forth a theoretical treatment which includes substrate interactions ignored by the Cohan-Kelvin theory. A brief outline of their capillary pore filling theory, including the influence of $\mathrm{vdW}$ interactions with the pore walls, is given here. We further simplify the theory by using the Derjaguin approximation [26] with the assumption that the capillary transition occurs when the thickness of the adsorbed liquid on the pore walls is small compared to the pore radius.

The total free energy per unit length of a cylindrically symmetric film on the walls of the pore is

$$
G(d)=2 \pi \gamma(R-d)+n \Delta \mu \pi\left(2 R d-d^{2}\right)+U(d),
$$

where $U(d)$ is the vdW potential between the liquid and the pore walls. For films thin relative to the pore radius, $d \ll$ $R, U(d)$ has the form equivalent to that for a liquid film on a flat substrate (Derjaguin approximation) [26]:

$$
U(d)=\frac{A_{\text {eff }}}{12 \pi d^{2}} 2 \pi R,
$$

where $A_{\text {eff }}=6.3 \times 10^{-20} \mathrm{~J}$ is the effective Hamaker constant for PFMC on $\mathrm{Al}_{2} \mathrm{O}_{3}$ [27]. In the limit of vanishing film thickness, this term should be replaced by the interfacial tension term for the pore wall-liquid interface. The equilibrium condition is then given by

$$
\begin{gathered}
\frac{\partial G(d)}{\partial N}=0 \\
\Delta \mu(d)=\frac{A_{\text {eff }}}{6 \pi n d^{3}}+\frac{\gamma}{n(R-d)}
\end{gathered}
$$

Equation (7) simplifies to the Kelvin equation when vdW interactions are ignored. Using Eq. (7), we fit the pretransition filling data for the absorption cycle, yielding a value of $R=11.8 \pm 0.2 \mathrm{~nm}$ (see Fig. 3 solid curve) in agreement with the Kelvin value obtained earlier. Discrepancies with the fit at large $\Delta T$ are possibly due to the film thickness being on the order of the monolayer thickness. The capillary transition to filled pores occurs when the chemical potential reaches the minimum allowed value, since further reduction of the chemical potential can only be achieved by complete filling of the pore, $d=R$, in which case the surface tension term vanishes due to the vanishing of the interface. This stability or critical condition for the cylindrically symmetric film is satisfied for $\partial \Delta \mu(d) / \partial N \geq 0$ (in the limit $d \ll R$ ):

$$
\frac{A_{\mathrm{eff}}}{2 \pi d^{4}} \geq \frac{\gamma}{(R-d)^{2}} \approx \frac{\gamma}{R^{2}}
$$

This demonstrates that the thin film is stabilized by the $\mathrm{vdW}$ interactions with the pore wall and that, at the transition, $d \approx 3.3 \mathrm{~nm}$, validating the use of the Derjaguin approximation, $d \ll R$.

For our system with $R=11.8 \mathrm{~nm}$, numerical calculations of Cole and Saam predict an onset of instability for volume fraction $V_{c} \approx 0.44$ (see Fig. 3 in Ref. [25]) assuming $R_{0}=\sqrt{A_{\text {eff }} / 2 \gamma} \approx 1.6 \mathrm{~nm}$ for the PFMC $/ \mathrm{Al}_{2} \mathrm{O}_{3}$ system. The estimate for the onset of the capillary transition, $V_{c}$ occurs at slightly higher $\Delta T$ than expected from the Kelvin and Derjaguin methods (see Fig. 3), but within the 
uncertainty given above. The above analysis indicates that for systems with ideal capillary geometry, such as the porous anodized alumina system studied here, the theory of Cole and Saam provides a good description of the capillary filling transition $\Delta T$.

Cole and Saam additionally make a prediction about the hysteresis upon desorption. They argue that the hysteresis occurs due to a partially filled pore with meniscus [see Fig. 2(c) (2)] being metastable with respect to a conformal thin film geometry [see Fig. 2(c) (1)]. The metastable limit is also a function of the pore radius, $R$, and $R_{0}$. The numerical analysis of Cole and Saam predicts a metastable volume fraction $V_{m} \approx 0.23$ for our experimental parameters (see Fig. 3 in Ref. [25]). The $V_{m}$ value approximately corresponds to the volume fraction where the desorption curve joins with the absorption curve, in good agreement with our experimental results (Fig. 3). It is worth noting that this joining occurs at $\Delta T \approx 2 \Delta T^{*}$, where the Cohan argument predicts pores to empty by hemispherical menisci. Unfortunately, there is no prediction of the $\Delta T$ where the desorption transition initiates, and it not possible to translate $V_{c}$ and $V_{m}$ into a hysteresis width.

For the cylindrical pores described here, the Kelvin equation is a reasonable predictor of the filling transition as well, though it must become increasingly worse for smaller pore size. However, the Kelvin-Cohan argument fails to qualitatively predict the observed hysteresis between adsorption and desorption. Cole and Saam's theory, which takes into account vdW interactions, both demonstrates good agreement with the experimental results in the prediction of the capillary transition and yields information about the observed hysteresis, yet there is not a satisfactory prediction of the observed width. It is clear from the experimental data that the desorption transition occurs at lower $\Delta T$ than either prediction. While some of the shift may be due to broadening of the transition by pore diameter polydispersity, this effect seems insufficient to fully account for the shift. Large pore aspect ratios rule out the influence of end effects and suggests other mechanisms for desorption are required to explain this feature of the data.

We are grateful to M. W. Cole for helpful discussions and comments. This work was supported by the National Science Foundation Grants No. 03-03916 and No. 0124936, along with Department of Energy Grant No. DEAC02-98CH10886 through the Nanoscale Science, Engineering, and Technology program at Brookhaven National Laboratory.

*Electronic address: kalvine@post.harvard.edu Current Address: National Institute of Standards and Technology, Gaithersburg, MD 20899 (USA)

[1] A. Meller, L. Nivon, and D. Branton, Phys. Rev. Lett. 86, 3435 (2001).
[2] Y. Rabin and M. Tanaka, Phys. Rev. Lett. 94, 148103 (2005).

[3] R. Karnik, R. Fan, M. Yue, D. Y. Li, P. D. Yang, and A. Majumdar, Nano Lett. 5, 943 (2005).

[4] K. J. Alvine, D. Pontoni, O. G. Shpyrko, P. S. Pershan, D. J. Cookson, K. Shin, T.P. Russell, M. Brunnbauer, F. Stellacci, and O. Gang, Phys. Rev. B 73, 125412 (2006); K. J. Alvine, Ph.D. thesis, Harvard University, 2006.

[5] M. Lahav, T. Sehayek, A. Vaskevich, and I. Rubinstein, Angew. Chem., Int. Ed. Engl. 42, 5576 (2003).

[6] R. E. Benfield, D. Grandjean, M. Kröll, R. Pugin, T. Sawitowski, and G. Schmid, J. Phys. Chem. B 105, 1961 (2001).

[7] J. P. Novak, E. S. Snow, E. J. Houser, D. Park, J. L. Stepnowski, and R. A. McGill, Appl. Phys. Lett. 83, 4026 (2003).

[8] O. K. Varghese, D. Gong, M. Paulose, K. G. Ong, C. A. Grimes, and E. C. Dickey, J. Mater. Res. 17, 1162 (2002).

[9] N. Maeda, J. N. Israelachvili, and M. M. Kohonen, Proc. Natl. Acad. Sci. U.S.A. 100, 803 (2003).

[10] M. M. Kohonen, N. Maeda, and H. K. Christenson, Phys. Rev. Lett. 82, 4667 (1999).

[11] H. K. Christenson, Phys. Rev. Lett. 73, 1821 (1994).

[12] L. D. Gelb and K. E. Gubbins, Langmuir 14, 2097 (1998).

[13] A. V. Neimark, and P. I. Ravikovitch, Microporous Mesoporous Mater. 44, 697 (2001).

[14] H. Masuda and K. Fukuda, Science 268, 1466 (1995).

[15] I. M. Tidswell, T. A. Rabedeau, P. S. Pershan, and S. D. Kosowsky, Phys. Rev. Lett. 66, 2108 (1991).

[16] O. Gang, K. J. Alvine, M. Fukuto, P. S. Pershan, C. T. Black, and B. M. Ocko, Phys. Rev. Lett. 95, 217801 (2005).

[17] K. Shin, H. Xiang, S. I. Moon, T. Kim, T. J. McCarthy, and T. P. Russell, Science 306, 76 (2004).

[18] R. K. Heilmann, M. Fukuto, and P.S. Pershan, Phys. Rev. B 63, 205405 (2001).

[19] W. D. Good, D. R. Douslin, D. W. Scott, A. George, J. L. Lacina, J. P. Dawson, and G. Waddington, J. Phys. Chem. 63, 1133 (1959).

[20] M. Deutsch, J. Appl. Crystallogr. 13, 252 (1980).

[21] J. Als-Nielsen and D. McMorrow, Elements of Modern X-Ray Physics (Wiley, New York, USA, 2001) p. 200.

[22] A. W. Adamson and A.P. Gast, Physical Chemistry of Surfaces (Wiley Interscience, New York, 1997), 6th ed. p. 665 .

[23] L. H. Cohan, J. Am. Chem. Soc. 60, 433 (1938).

[24] M. W. Cole and W.F. Saam, Phys. Rev. Lett. 32, 985 (1974).

[25] W.F. Saam and M. W. Cole, Phys. Rev. B 11, 1086 (1975).

[26] B. V. Derjaguin, Acta Physicochim. URSS 12, 181 (1940).

[27] This value for $A_{\text {eff }}$ for PFMC on $\mathrm{Al}_{2} \mathrm{O}_{3}$ relies on the approximation $A_{12} \approx \sqrt{A_{11} A_{22}}$ (see Ref. [28]) and the values $A_{11}(\mathrm{PFMC})=2.8 \times 10^{-20} \mathrm{~J}$ (see Ref. [18]) and $A_{11}\left(\mathrm{Al}_{2} \mathrm{O}_{3}\right)=14 \times 10^{-20} \mathrm{~J}$ (see Ref. [28]).

[28] J. N. Israelachvili, Intermolecular \& Surface Forces (Academic Press, New York, 1992), 2nd. ed. 